\title{
A Three-Scale Finite Element Method for Elliptic Equations with Rapidly Oscillating Periodic Coefficients
}

\author{
Henrique Versieux ${ }^{1}$ and Marcus Sarkis ${ }^{2}$ \\ 1 Instituto Nacional de Matemática Pura e Aplicada, Rio de Janeiro, Brasil. \\ versieux@impa.br \\ 2 msarkis@impa.br
}

\section{Introduction}

On several real world problems the scale $\epsilon$ is so smaller than $\Omega$ that even with very heavy computer efforts it is impossible to take $h<\epsilon, h$ being the scale (mesh-size) of the discrete method used to approximate the solution of

$$
L_{\epsilon} u_{\epsilon}=-\frac{\partial}{\partial x_{i}}\left(a_{i j}(x / \epsilon) \frac{\partial}{\partial x_{j}} u_{\epsilon}=f \text { in } \Omega, u_{\epsilon}=0 \text { on } \partial \Omega .\right.
$$

where the matrix $a(y)=\left(a_{i j}(y)\right)$ is symmetric positive definite, whose entries are periodic functions of $y$ with periodic cell $Y$. More specifically we assume $a_{i j} \in C^{1, \beta}\left(\Re^{2}\right), \beta>0$. It is also assumed that there exists positive constants $\gamma_{a}$ and $\beta_{a}$ such that $\gamma_{a}\|\xi\|^{2} \leq a_{i j}(y) \xi_{i} \xi_{j} \leq \beta_{a}\|\xi\|^{2}$ for all $\xi \in \Re^{2}$ and $y \in \bar{Y}$. Recently new numerical methods have been proposed for approximating the solution $u_{\epsilon}$ with meshes sizes $h>\epsilon$ (or $h>>\epsilon$ ) but capturing the oscillations presented by the the solution $u_{\epsilon}$; see for example [HW97,EHW00,SM02,EE03,S03,AB04,]. In [VS05a] we developed a numerical scheme for this problem for the case the domain $\Omega$ is rectangular, and quasi-optimal error rate estimates were obtained. That method, opposed to the methods [HW97,EHW00,S03] is strongly based on asymptotic expansions of $u_{\epsilon}$. We construct a first order asymptotic expansion for $u_{\epsilon}$, and then we numerically approximate each term separately.

In this paper, we modify the method in [VS05a] for the case where $\Omega$ is a convex polygonal regional with rational normals. In this case, a better treatment for the normal derivative of $u_{0}$ is required. We propose an approximation based on hybrid finite element for the flux and we obtain optimal error rate estimates for the $L_{2}$ norm and $H^{1}$ broken semi-norm. 


\section{Notation}

We assume that $Y=[0,1] \times[0,1]$ and $\Omega$ is bounded convex polygonal region in $\Re^{2}$, whose boundary $\partial \Omega=\cup \Gamma^{k}, k=1, \ldots, m$ where each $\Gamma^{k}$ is a line segment with minimal outward normal denoted by $N_{k}=\left(p_{k}, q_{k}\right)^{t}$, where $p_{k}$ and $q_{k}$ are integers and relative primes. This hypothesis is required to guarantee periodicity of $a(x / \epsilon)$ on $\Gamma_{k}$ [MV97].

Let $D \subset \Re^{2}$ be an open set. We use the standard notation $\|\cdot\|_{s, D},\|\cdot\|_{s, p, D}$ for $H^{s}(D)$ and $W_{p}^{s}(D)$ norms, $|\cdot|_{s, D},|\cdot|_{s, p, D}$ their semi-norms. and $\|\cdot\|_{s, h, D}$ for the broken norms related to a regular partition $\mathcal{T}_{h}(D)=K_{1}, K_{2}, \ldots, K_{m}$ of $D$. Throughout this paper, when we do not make reference to the domain $D$ it is assumed that $D=\Omega$. It is continually used the Einstein summation convention, i.e. repeated indices indicate summation, except when the indice $k$ is used. In what follows $c$ denotes a generic constant independent of $\epsilon, h$, and functions being evaluated.

\section{Theoretical Approximation}

\subsection{The Asymptotic Expansion}

The solution $u_{\epsilon}$ can be approximated by an asymptotic expansion. This approximation can be found using equation (1) and the ansatz

$$
u_{\epsilon}(x)=u_{0}(x, x / \epsilon)+\epsilon u_{1}(x, x / \epsilon)+\epsilon^{2} u_{2}(x, x / \epsilon)+\cdots,
$$

where the functions $u_{j}(x, y)$ are $Y$ periodic in $\mathrm{y}$. These terms are defined below; for more details see [BLP80,OSY92,MV97] .

Let $\chi^{j}$ be the $Y$ periodic solution with zero average on $Y$ of

$$
\nabla_{y} \cdot a(y) \nabla_{y} \chi^{j}=\nabla_{y} \cdot a(y) \nabla_{y} y_{j}=\frac{\partial}{\partial y_{i}} a_{i j}(y) .
$$

We have that $\chi^{j} \in C^{2, \beta}\left(\Re^{2}\right)$ when $a_{i j} \in C^{1, \beta}\left(\Re^{2}\right)$. Define the matrix:

$$
A_{i j}=\frac{1}{|Y|} \int_{Y} a_{l m}(y) \frac{\partial}{\partial y_{l}}\left(y_{i}-\chi^{i}\right) \frac{\partial}{\partial y_{m}}\left(y_{j}-\chi^{j}\right) d y .
$$

It is easy to see that the matrix $A$ is symmetric positive definite. Define $u_{0} \in H^{2}(\Omega) \cap H_{0}^{1}(\Omega)$ as the solution of

$$
-\nabla \cdot A \nabla u_{0}=f \text { in } \Omega, \quad u_{0}=0 \text { on } \partial \Omega,
$$

and let $u_{1}\left(x, \frac{x}{\epsilon}\right)=-\chi^{j}\left(\frac{x}{\epsilon}\right) \frac{\partial u_{0}}{\partial x_{j}}(x)$. Note that $u_{0}+\epsilon u_{1}$ does not satisfy the zero Dirichlet boundary condition on $\partial \Omega$. In order to correct this, the boundary corrector term $\theta_{\epsilon} \in H^{1}(\Omega)$ is introduced as the solution of

$$
-\nabla \cdot a(x / \epsilon) \nabla \theta_{\epsilon}=0 \text { in } \Omega, \quad \theta_{\epsilon}=-u_{1}\left(x, \frac{x}{\epsilon}\right) \text { on } \partial \Omega .
$$

Therefore we obtain $u_{0}+\epsilon u_{1}+\epsilon \theta_{\epsilon} \in H_{0}^{1}(\Omega)$. 


\subsection{Boundary Corrector Approximation}

Note that the coefficients $a_{i j}(x / \epsilon)$ and the boundary values $-u_{1}\left(x, \frac{x}{\epsilon}\right)$ of the Equation (5) are highly oscillatory, hence it is not a trivial problem to obtain a good discretization for $\theta_{\epsilon}$. We propose an analytical approximation for $\theta_{\epsilon}$, denoted by $\phi_{\epsilon}$ that satisfies the oscillating boundary condition and is more suitable for numerical approximation.

Note that $u_{0}=0$ along $\partial \Omega$ implies $\left.\nabla u_{\epsilon}\right|_{\Gamma_{k}}=\eta_{k} \partial_{\eta_{k}} u_{0}$. We then decompose $\theta_{\epsilon}=\tilde{\theta}_{\epsilon}+\bar{\theta}_{\epsilon}$ where

$$
-\nabla \cdot a(x / \epsilon) \nabla \tilde{\theta}_{\epsilon}=0 \text { in } \Omega, \quad \tilde{\theta}_{\epsilon}=-u_{1}-\chi^{*} \partial_{\eta} u_{0} \text { on } \partial \Omega,
$$

and

$$
-\nabla \cdot a(x / \epsilon) \nabla \bar{\theta}_{\epsilon}=0 \text { in } \Omega, \quad \bar{\theta}_{\epsilon}=\chi^{*} \partial_{\eta} u_{0} \text { on } \partial \Omega,
$$

where $\left.\chi^{*}\right|_{\Gamma_{k}}=\chi_{k}^{*}$ are properly chosen constants . In Remark 1 we show that the problems (6) and (7) are well posed. The approximation $\phi_{\epsilon}$ for $\theta_{\epsilon}$ is defined later as $\tilde{\phi}_{\epsilon}+\bar{\phi}_{\epsilon}$, where $\tilde{\phi}_{\epsilon} \approx \tilde{\theta}_{\epsilon}$ and $\bar{\phi}_{\epsilon} \approx \bar{\theta}_{\epsilon}$.

Next we define constants $\chi_{k}^{*}$ for which the approximation $\tilde{\phi}_{\epsilon}$ decays exponentially to zero away from the boundary and is suitable for numerical approximation.

Let $\tau_{k}=\left(\eta^{k}\right)^{\perp}$ be the $\pi / 2$ rotation counterclockwise of $\eta^{k}$. We introduce the following normal and tangential coordinate system

$$
\left(\begin{array}{l}
y_{1}^{\prime} \\
y_{2}^{\prime}
\end{array}\right)=-\left(\begin{array}{c}
\eta^{k^{T}} y \\
\tau_{k}^{T} y
\end{array}\right)
$$

We observe that a function periodic in $y$ with period 1 is periodic in $y^{\prime}$ with period $T_{k}=\left(p_{k}^{2}+q_{k}^{2}\right)^{1 / 2}$. Associated to each side $\Gamma_{k}$ of $\partial \Omega$, let $G_{k}=\{y \in$ $R^{2} ; y_{1}^{\prime} \leq 0 ;$ and $\left.0 \leq y_{2}^{\prime} \leq T_{k}\right\}$; and $v_{k}$ the solution of

$$
\begin{aligned}
& -\nabla_{y} \cdot a\left(y+\delta_{\epsilon} \eta^{k}\right) \nabla_{y} v_{k}=0 \text { in } G_{k}, \\
& v_{k}(y)=\chi^{j}\left(y+\delta_{\epsilon} \eta^{k}\right) \eta_{j}^{k} \quad \text { on }\left\{y \in G_{k}, y_{1}^{\prime}=0\right\} \\
& \left.v_{k}\right|_{y_{2}^{\prime}=0}=\left.v_{k}\right|_{y_{2}^{\prime}=T_{k}}, \text { for } \quad-\infty<y_{1}^{\prime}<0, \\
& \text { and } \frac{\partial v_{k}}{\partial y_{i}} \exp \left(-\gamma y_{1}^{\prime}\right) \in L^{2}\left(G_{k}\right), \quad i=1,2,
\end{aligned}
$$

where $\delta_{\epsilon}=T_{k}\left(s_{k} /\left(\epsilon T_{k}\right)-\left\lfloor s_{k} /\left(\epsilon T_{k}\right)\right\rfloor\right)$, and $s_{k}$ is such that $\Gamma_{k} \subset\left\{x \in \Re^{2} ; x\right.$. $\left.\eta_{k}=s_{k}\right\} ;(\lfloor\cdot\rfloor$ denotes the integer part $)$.

Let

$$
\begin{aligned}
\chi_{k}^{*}= & \frac{1}{\left(A \eta^{k}, \eta^{k}\right) T_{k}}\left(\left.\int_{0}^{T_{k}}\left[\chi^{l} a_{i j}\left(\delta_{j m}-\frac{\partial \chi^{m}}{\partial y_{j}}\right) \eta_{i}^{k} \eta_{m}^{k} \eta_{l}^{k}\right]\right|_{y_{1}^{\prime}=\delta_{\epsilon}} d y_{2}^{\prime}\right. \\
& \left.+\int_{G_{k}}\left(a\left(y+\delta_{\epsilon} \eta^{k}\right) \nabla_{y} v_{k} \cdot \nabla_{y} v_{k}\right) d y\right)
\end{aligned}
$$

It can be shown [MV97] that $v_{k}$ decays exponentially to zero for $y_{1}^{\prime} \rightarrow-\infty$, i.e. $\left(v_{k}-\chi_{k}^{*}\right) \exp \left(-\gamma y_{1}^{\prime}\right) \in L^{2}\left(G_{k}\right)$. 
We note by Remark 1 that $\left.\left(u_{1}\left(x, \frac{x}{\epsilon}\right)-\chi^{*} \partial_{\eta} u_{0}\right)\right|_{\Gamma_{k}} \in H_{00}^{1 / 2}\left(\Gamma_{k}\right)$. Thus we can split $\tilde{\theta}_{\epsilon}=\sum_{k \in\{1, \ldots, N\}} \tilde{\theta}_{\epsilon}^{k}$, where

$$
L_{\epsilon} \tilde{\theta}_{\epsilon}^{k}=0 \quad \text { in } \Omega, \quad \tilde{\theta}_{\epsilon}^{k}= \begin{cases}-u_{1}\left(x, \frac{x}{\epsilon}\right)-\chi^{*} \partial_{\eta} u_{0} & \text { on } \Gamma_{k}, \\ 0 & \text { on } \partial \Omega \backslash \Gamma_{k} .\end{cases}
$$

We approximate $\tilde{\theta}_{\epsilon}^{k}$ by $\tilde{\phi}_{\epsilon}^{k}$ given by

$$
\tilde{\phi}_{\epsilon}^{k}\left(x_{1}, x_{2}\right)=\varphi_{k}\left(x_{1}\right)\left(v_{k}\left(\frac{x-s_{k} \eta_{k}}{\epsilon}\right)-\chi_{k}^{*}\right) \nabla u_{0} \cdot \eta_{k} .
$$

In order to simplify the definition of the function $\varphi_{k}(x)$ let us assume $\Gamma_{k}=\left\{x \in \Re^{2} ; x_{1}=0,0 \leq x_{2} \leq c\right\}$ and that $x_{1}^{+}$is the inner normal direction. Let $\Gamma_{k-1}, \Gamma_{k+1}$ be the edges with vertices at the point $(0, c),(0,0)$ respectively and let $\alpha_{k}>0$ and $\alpha_{k+1}<0$ be the angles between $x_{1}$ axis and $\Gamma_{k-1}$ and $\Gamma_{k+1}$ respectively. Then we define

$$
\varphi_{k}(x)= \begin{cases}1 & \text { if } 0 \leq x_{1} \leq \delta ; 0 \leq x_{2} \leq c \\ 1-\left(x_{2}-c\right) /\left(x_{1} \tan \alpha_{k}\right) & \text { if } 0 \leq x_{1} \leq \delta ; x_{2}>c \\ 1+x_{2} /\left(x_{1} \tan \alpha_{k+1}\right) & \text { if } 0 \leq x_{1} \leq \delta ; x_{2}<0 \\ \text { smooth } & \text { if } \delta \leq x_{1} \leq 2 \delta \\ 0 & \text { if } x_{1} \geq 2 \delta\end{cases}
$$

Hence $\tilde{\phi}_{\epsilon}=\sum_{k \in\{1, \ldots, N\}} \tilde{\phi}_{\epsilon}^{k}$ approximate $\tilde{\theta}_{\epsilon}$, and $\tilde{\phi}_{\epsilon}=\tilde{\theta}_{\epsilon}$ on the boundary of $\Omega$.

The boundary condition imposed on Equation (7) does not depend on $\epsilon$. An effective approximation for $\bar{\theta}_{\epsilon}$ is given by $\bar{\phi} \in H^{1}(\Omega)$ the solution of

$$
-\nabla \cdot A \nabla \bar{\phi}=0 \text { in } \Omega, \quad \bar{\phi}=\chi^{*} \partial_{\eta} u_{0} \text { on } \partial \Omega .
$$

We define our theoretical approximation for $u_{\epsilon}$ as $u_{0}+\epsilon u_{1}+\epsilon \phi_{\epsilon}$, where $\phi_{\epsilon}=$ $\tilde{\phi}_{\epsilon}+\bar{\phi}$. Note that $\left.\phi_{\epsilon}\right|_{\partial \Omega}=\left.\theta_{\epsilon}\right|_{\partial \Omega}$, therefore $u_{0}+\epsilon u_{1}+\epsilon \phi_{\epsilon}=0$ on $\partial \Omega$. In [VS05b] we prove the following error bounds

Theorem 1. Assume that $a_{i j} \in C^{1, \beta}\left(\Re^{2}\right)$ and $u_{0} \in H^{2}(\Omega),\left(u_{0} \in H^{3}(\Omega)\right)$. Then there exists a constant $c$, such that

$$
\begin{aligned}
& \left\|u_{\epsilon}-u_{0}-\epsilon u_{1}-\epsilon \phi_{\epsilon}\right\|_{1} \leq c \epsilon\left\|u_{0}\right\|_{2} \\
& \left(\left\|u_{\epsilon}-u_{0}-\epsilon u_{1}-\epsilon \phi_{\epsilon}\right\|_{0} \leq \epsilon^{3 / 2}\left\|u_{0}\right\|_{3}\right) .
\end{aligned}
$$

Remark 1. Since $u_{0}$ satisfies zero Dirichlet boundary condition on $\partial \Omega$ and $u_{0} \in H^{2}(\Omega)$, we have $\frac{\partial u_{0}}{\partial \eta^{k}} \in H_{00}^{1 / 2}\left(\Gamma_{k}\right)$ and $\left\|\chi^{*} \partial_{\eta} u_{0}\right\|_{H^{1 / 2}(\partial \Omega)} \leq c\left(\chi^{*}\right)\left\|u_{0}\right\|_{2}$. Note also that $u_{1}\left(x, \frac{x}{\epsilon}\right)=-\chi^{j}\left(\frac{x}{\epsilon}\right) \frac{\partial u_{0}}{\partial x_{j}}(x)$, since $\chi^{j} \in C^{2, \beta}\left(\Re^{2}\right)$ we get $\left.u_{1}\right|_{\Gamma_{k}} \in$ $H_{00}^{1 / 2}\left(\Gamma_{k}\right)$. 


\section{Finite Element Approximation}

We now describe how to numerically approximate the terms $u_{0}, u_{1}, \tilde{\phi}_{\epsilon}$ and $\bar{\phi}$.

- Solve the cell problem (2) with a second order accurate conforming finite element in a partition $\mathcal{T}_{\hat{h}}(Y)$. Call these solutions $\chi_{\hat{h}}^{j}$.

- Define $A_{i j}^{\hat{h}}=\frac{1}{|Y|} \int_{Y} a_{l m}(y) \frac{\partial}{\partial y_{l}}\left(y_{i}-\chi_{\hat{h}}^{i}\right) \frac{\partial}{\partial y_{m}}\left(y_{j}-\chi_{\hat{h}}^{j}\right) d y$.

- Let $V^{h}(\Omega)$ be a conforming second order accurate finite element in a mesh $\mathcal{T}_{h}(\Omega)$, and $V_{0}^{h}(\Omega)=V^{h}(\Omega) \cap H_{0}^{1}(\Omega)$. Define $u_{0}^{h, \hat{h}} \in V_{0}^{h}$ the solution of

$$
\int_{\Omega}\left(A^{\hat{h}} \nabla u_{0}^{h, \hat{h}}, \nabla v^{h}\right) d x=\int_{\Omega} f v^{h} d x, \quad \forall v^{h} \in V_{0}^{h} .
$$

- Define $u_{1}^{h, \hat{h}}$ as $u_{1}^{h, \hat{h}}(x)=-\chi_{\hat{h}}^{j}\left(\frac{x}{\epsilon}\right) \frac{\partial u_{0}^{h, \hat{h}}}{\partial x_{j}}(x)$. Note that this leads to a nonconforming approximation for $u_{1}$ in the partition $\mathcal{T}_{h}(\Omega)$.

- Define $Y_{k}^{h}$ the trace of $V^{h}$ at $\Gamma_{k}$. And let $\lambda_{k}^{h} \in \Gamma_{k}^{h}, \lambda_{k}^{h}=0$ at $\partial \Gamma_{k}$ satisfying

$$
\int_{\Omega} A_{i j}^{\hat{h}} \partial_{i} u_{0}^{h} \partial_{j} \phi d x=\int_{\Omega} f \phi d x+\int_{\Gamma_{k}} \lambda_{k}^{h} \phi d \sigma .
$$

$\forall \phi \in V^{h} ;\left.\phi\right|_{\partial \Omega \backslash \Gamma_{k}}=0$ so approximate $\partial_{\eta} u_{0}$ by $\mu^{h, \hat{h}}$ where

$$
\left.\mu^{h, \hat{h}}\right|_{\Gamma_{k}}=\lambda_{k}^{h} / A_{l l}^{\hat{h}}, \quad\left\{\begin{array}{l}
l=1 \text { if } k=1,3 . \\
l=2 \text { if } k=2,4 .
\end{array}\right.
$$

- Let $p$ be a positive integer and $G_{k}^{p}=\left\{y \in R^{2} ; y_{1}^{\prime} \leq 0,\left|y_{1}^{\prime}\right| \leq p\right.$; and $0 \leq$ $\left.y_{2}^{\prime} \leq T_{k}\right\}$. Define $\tilde{v}_{k} \in H^{1}\left(G_{k}^{p}\right)$ the solution of

$$
\begin{aligned}
& -\nabla_{y} \cdot a\left(y+\delta_{\epsilon} \eta^{k}\right) \nabla_{y} \tilde{v}_{k}=0 \text { in } G_{k}^{p}, \\
& \tilde{v}_{k}(y)=\chi_{\hat{h}}^{1}\left(y+\delta_{\epsilon} \eta^{k}\right), \text { on }\left\{y \in G_{k}, y_{1}^{\prime}=0\right\}, \\
& \partial_{\eta} \tilde{v}_{k}=0, \text { on }\left\{y \in G_{k}^{p} ;\left|y_{1}^{\prime}\right|=p\right\}, \\
& \text { and }\left.v_{k}\right|_{y_{2}^{\prime}=0}=\left.v_{k}\right|_{y_{2}^{\prime}=T_{k}}, \text { for }\left|y_{1}^{\prime}\right|<p .
\end{aligned}
$$

Let $v_{k}^{\hat{h}, p}$ be a numerical approximation of $\tilde{v}_{k}$ using a second order accurate conforming finite element on a mesh $\mathcal{T}_{\hat{h}}\left(G_{e}^{p}\right)$.

- Define

$$
\begin{aligned}
\chi_{k}^{*, \hat{h}, p}= & \frac{1}{\left(A^{\hat{h}} \eta^{k}, \eta^{k}\right) T_{k}}\left(\left.\int_{0}^{T_{k}}\left[\chi_{\hat{h}}^{l} a_{i j}\left(\delta_{j m}-\frac{\partial \chi_{\hat{h}}^{m}}{\partial y_{j}}\right) \eta_{i}^{k} \eta_{m}^{k} \eta_{l}^{k}\right]\right|_{y_{1}^{\prime}=\delta_{\epsilon}} d y_{2}^{\prime}\right. \\
& \left.+\int_{G_{k}}\left(a\left(y+\delta_{\epsilon} \eta^{k}\right) \nabla_{y} v_{k}^{\hat{h}, p} \cdot \nabla_{y} v_{k}^{\hat{h}, p}\right) d y\right)
\end{aligned}
$$

- Given $g: \Gamma_{k} \rightarrow \Re$, let $E_{k}(g) \in V^{h}(\Omega)$ be the extension by zero of $g$ to $\Omega$. 
- Observe that in Equation. (9) the term $v_{k}\left(\left(x-s_{k} \eta_{k}\right) / \epsilon\right)$ appears. Since the approximation $v_{k}^{\hat{h}, p}$ is defined in $G_{k}^{p}$, we can calculate $v_{k}^{\hat{h}, p}\left(\left(x-s_{k} \eta_{k}\right) / \epsilon\right)$ only if $\left|x_{1}^{\prime}-s_{k}\right| \leq \epsilon p$. Since the functions $v_{k}-\chi_{k}^{*}$ decays exponentially to zero in the $-\eta_{k}$ direction its is natural to consider the following approximation

$$
\begin{aligned}
& \tilde{\phi}_{\epsilon}^{e, h, \hat{h}, p}\left(x_{1}, x_{2}\right)= \\
& \begin{cases}\varphi_{k}\left(v_{k}^{\hat{h}, p}\left(\frac{x-s_{k} \eta_{k}}{\epsilon}\right) \frac{\partial u_{0}^{h, \hat{h}}}{\partial x_{1}}-\chi_{k}^{*, \hat{h}, p} E_{k}\left(\mu^{h, \hat{h}}\right)\right) & \text { if }\left|x_{1}^{\prime}-s_{k}\right|<\epsilon p, \\
0 & \text { if }\left|x_{1}^{\prime}-s_{k}\right| \geq \epsilon p,\end{cases}
\end{aligned}
$$

and $\tilde{\phi}_{\epsilon}^{h, \hat{h}, p}=\sum_{k \in\{1, \ldots, N\}} \tilde{\phi}_{\epsilon}^{k, h, \hat{h}, p}$.

- Let $\bar{\phi}^{h, \hat{h}, p}$ be a second order accurate finite element approximation in a mesh of size $h$ for the following equation

$$
-\nabla A^{\hat{h}} \nabla \psi=0, \quad \psi=\chi^{*, \hat{h}, p} \mu^{h, \hat{h}} \text { on } \partial \Omega .
$$

Remark 2. By construction $\mu^{h, \hat{h}}=0$ at the corners of $\Omega$, therefore $\chi^{*} \mu^{h, \hat{h}} \in H^{1 / 2}(\partial \Omega)$. This implies that Eq.(11) is well posed. In addition $\left.\chi^{*} \mu^{h, \hat{h}} \in V^{h}\right|_{\partial \Omega}$ hence we can look for a numerical solution of Eq.(11) at $V^{h}$.

- Approximate $\theta_{\epsilon}$ by $\theta_{\epsilon}^{h, \hat{h}, p}:=\phi^{r, h, \hat{h}, p}+\theta^{*, h, \hat{h}, p}$ and finally construct the numerical solution for Eq. (1), $u_{\epsilon}^{h, \hat{h}, p}=u_{0}^{h, \hat{h}}+\epsilon u_{1}^{h, \hat{h}}+\epsilon \theta_{\epsilon}^{h, \hat{h}, p}$.

\section{Error Analysis}

When $p \rightarrow \infty$ and $\hat{h} \rightarrow 0$ we prove in [VS05b] the following estimates.

Theorem 2. Assume that $a_{i j} \in C^{1, \beta}\left(\Re^{2}\right)$ and $u_{0} \in W^{2, \infty}(\Omega) \quad\left(u_{0} \in\right.$ $\left.W^{2, \infty}(\Omega) \cap H^{3}(\Omega)\right)$. Then there exists a constant $c$, such that

$$
\begin{gathered}
\left|u_{\epsilon}-u_{h}\right|_{1, h} \leq c(h+\epsilon)\left\|u_{0}\right\|_{2, \infty} \\
\left(\left\|u_{\epsilon}-u_{h}\right\|_{0} \leq c\left(h^{2}+\epsilon^{\frac{3}{2}}+\epsilon h\right)\left(\left|u_{0}\right|_{2, \infty}+\left\|u_{0}\right\|_{3}\right)\right)
\end{gathered}
$$

\section{Numerical Experiments}

In this section, we present some numerical results for solving our model problem with

$$
a(x)=\left(\frac{2+P \sin \left(2 \pi x_{1} / \epsilon\right)}{2+P \cos \left(2 \pi x_{2} / \epsilon\right)}+\frac{2+\sin \left(2 \pi x_{2} / \epsilon\right)}{2+P \sin \left(2 \pi x_{1} / \epsilon\right)}\right) I_{2 \times 2}
$$




$$
f(x)=-1 \quad \text { and } \quad u=0 \text { on } \partial \Omega .
$$

We compare the solution obtained by our method with the solution obtained by a second order accurate finite element method in a fine mesh of size $h_{f}$, which we call $u_{\epsilon}^{*}$. Tables 1 provide absolute errors estimates for $u_{\epsilon}^{*}-u_{\epsilon}^{h, \hat{h}, p}$. We have used $p=2, \hat{h}=1 / 128, h_{f}=1 / 2048$, and a triangular mesh with continuous piecewise linear functions to approximate $\chi_{\hat{h}}^{j}$ and $v_{k}^{\hat{h}, p}$. From Table

Table 1. $u_{\epsilon}^{*}-u_{\epsilon}^{h, \hat{h}, p}$ error

\begin{tabular}{l}
\multicolumn{7}{c}{$\|\cdot\|_{0}$ error } \\
\begin{tabular}{|l|l|l|l|l|}
\hline$\epsilon \downarrow \quad h \rightarrow$ & $1 / 8$ & $1 / 16$ & $1 / 32$ & $1 / 64$ \\
\hline $1 / 16$ & $2.3863 \mathrm{e}-04$ & $1.5793 \mathrm{e}-04$ & & \\
\hline $1 / 32$ & $2.3241 \mathrm{e}-04$ & $8.0169 \mathrm{e}-05$ & $1.7773 \mathrm{e}-05$ & \\
\hline $1 / 64$ & $2.3540 \mathrm{e}-04$ & $5.4314 \mathrm{e}-05$ & $5.1865 \mathrm{e}-05$ & $5.9606 \mathrm{e}-05$ \\
\hline \multicolumn{5}{|c|}{$|\cdot| 1, h$ error } \\
\hline $1 / 16$ & 0.0097 & 0.0067 & & \\
\hline $1 / 32$ & 0.0086 & 0.0051 & 0.0036 & \\
\hline $1 / 64$ & 0.0086 & 0.0044 & 0.0025 & 0.0018 \\
\hline
\end{tabular}
\end{tabular}

Table 2.

\begin{tabular}{|l|l||l|}
\hline \multicolumn{3}{|c}{$\epsilon=1 / 64, h=1 / 32, h_{f}=1 / 1024$} \\
\hline$u_{\epsilon}^{*}-u_{0}^{h, \hat{h}}$ & $\|\cdot\|_{0}$ & $\left.\cdot\right|_{1, h}$ \\
\hline$u_{\epsilon}^{*}-u_{0}^{h, \hat{h}}-\epsilon u_{1}^{h, \hat{h}}$ & 0.0287 & 0.0215 \\
\hline$u_{\epsilon}^{*}-u_{0}^{h, \hat{h}}-\epsilon u_{1}^{h, \hat{h}}-\epsilon \bar{\phi}^{h, \hat{h}, p}$ & 0.0213 & 0.0026 \\
\hline$u_{\epsilon}^{*}-u_{0}^{h, \hat{h}}-\epsilon u_{1}^{h, \hat{h}}-\epsilon\left(\bar{\phi}^{h, \hat{h}, p}+\tilde{\phi}_{\epsilon}^{h, \hat{h}, p}\right)$ & $5.0450 \mathrm{e}-05$ & 0.0026 \\
\hline
\end{tabular}

1, we see that for $\epsilon<<h$ we have errors of order $O\left(h^{2}\right)$ and $O(h)$ for the $L^{2}$ norm and semi norm $H^{1}$ respectively. We observe that when we fix $h$ and decrease $\epsilon$ the errors almost do not change. This is an evidence that in this case the dominant error term is $O(h)$. Also looking the diagonal values in these tables we see clearly that the numerical error agrees with the theoretical rates from Theorem 2.

Table 2 shows the improvement obtained in the final approximation by considering the numerical approximation for the boundary corrector. We observe a better improvement on the $\|\cdot\|_{0}$ norm rather then on $|\cdot|_{1, h}$ semi norm. The reason for this is that $\bar{\phi}$ is obtained through the homogenized equation associated to Problem (7), therefore it is a good approximation for $\bar{\theta}_{\epsilon}$ on $L^{2}(\Omega)$ norm but not on $|\cdot|_{1}$ semi norm. The term $\tilde{\phi}_{\epsilon}$ is defined in a thin boundary layer that mostly force the approximation to satisfies the zero Dirichlet boundary condition. 


\section{Conclusions}

We propose a new method for approximating numerically the solution of Equation (1). This method is strongly based on periodicity of the coefficients $a_{i j}$, and for this reason it has relative low computational cost with optimal error convergence rate.

\section{References}

[AB04] Allaire G., Brizzi R.: A multiscale finite element method for numerical homogenization. Internal report, CMAP, Ecole Polytechnique, 545 (2004).

[BLP80] Bensoussan A., Lions JL., Papanicolaou G.: Asymptotic Analysis for Periodic Structures. North Holland, (1980)

[EE03] E W, Engquist B. The heterogeneous multiscale method. Comm. Math. Sci., 1, 87-132 (2003)

[EHW00] Efendiev Y.R., Hou T., Wu X.H. . Convergence of nonconforming multiscale finite element method. SIAM J. Numer Anal, 37: 888-910, (2000)

[HW97] Hou T, Wu XH. A Multi-scale finite element method for elliptic problems in composite materials and porous media. J. of Comp. Phys, 134, 169-189 (1997).

[HWC99] Hou T, Wu XH, Cai Z. Convergence of multi-scale finite element method for elliptic problems with rapidly oscillating coefficients. Mathematics of Computation, 68, 913-943 (1999)

[GR85] Grisvard P. Elliptic Problems in Nonsmooth Domains Pitman, (1985)

[LI87] P.L. Lions On the Schawarz Alternating Method. I Proceedings of the First DD meeting in Paris, (1987)

[MV97] Moscow S, Vogelius M. First-order corrections to the homogenized eigenvalues of a periodic composite medium. A convergence proof. Proceedings of the Royal Society of Edinburgh, 127A, 1263-1299 (1997)

[OSY92] Oleinik O, Shamev AS, Yosifian GA. Mathematical Problems in Elasticity and Homogenization, North-Holland, Amsterdam (1992)

[PE88] P. Peisker, On the Numerical Solution of the First Biharmonic Equation", RAIRO Math. Model Num Analysis 22 655-676 (1988)

[S03] Sangalli G. Capturing small scales in elliptic problems using a residualfree bubbles finite element method. SIAM Multi-scale Model. Simul., 1, 485-503 (2003)

[SM02] Schwab C, Matache AM. Generalized FEM for Homogenization Problems. Lecture Notes in Computational Science and Engineering, Springer (2002)

[VS05a] Versieux HM, Sarkis M. Numerical boundary correctors for elliptic equations with Rapidly Oscillating Periodic Coefficients. Submitted to Comunications on Numerical Methods in Engineering

[SV05b] Versieux HM., Sarkis M, Convergence analysis of numerical boundary correctors for elliptic equations with Rapidly Oscillating Periodic Coefficients. Paper in preparation 